\title{
Total aortic arch reconstruction with open placement of triple-branched stent graft for acute type A dissection
}

\author{
Liang-Wan Chen, MD, Xiao-Fu Dai, MD, Gui-Can Zhang, MD, and Lin Lu, MD, Fuzhou, Fujian, China
}

In total arch replacement for acute type A aortic dissection, careful manipulations of the arch and elaborate anastomoses to the distal aortic and three arch vessels are time-consuming and could induce phrenic and recurrent laryngeal nerve injury. ${ }^{1,2}$ Moreover, performing anastomosis and hemostasis at the descending aorta and at the left subclavian artery is usually very difficult because of the deep surgical field. ${ }^{1,3}$ To reduce such problems, we applied a simple open triplebranched stent graft placement technique for total arch reconstruction.

\section{CLINICAL SUMMARY}

From June 2008 to May 2009, the triple-branched stent graft was used in 5 selective patients ( 4 men and 1 woman; mean age, 60 years; range, 56-65 years). All had acute DeBakey type I aortic dissection with the entry site in the ascending aorta. There were no dissection-related complications.

A median sternotomy was performed and cardiopulmonary bypass was established by two venous cannulas through the right atrium and two arterial return cannulas placed into the femoral and right axillary arteries. After core cooling to a $22^{\circ} \mathrm{C}$ rectal temperature, selective cerebral perfusion via the right axillary artery was established and perfusion to the lower body was discontinued. After we crossclamped the left common carotid artery $(4 \mathrm{~cm}$ above the arch) and innominate artery $(5 \mathrm{~cm}$ above the arch), the ascending aorta was transected at the base of the innominate artery. Through the transverse incision, the main graft of the triple-branched stent graft (Yuhengjia Sci \& Tech Corp Ltd, Beijing, China; Figure 1) was inserted into the true lumen of the arch and descending aorta, and then each sidearm graft

From the Department of Cardiac Surgery, Union Hospital, Fujian Medical University, Fuzhou, Fujian, China.

Disclosures: None.

Received for publication Sept 11, 2009; revisions received Oct 5, 2009; accepted for publication Oct 14, 2009; available ahead of print Jan 14, 2010.

Address for reprints: Liang-Wan Chen, MD, Department of Cardiac Surgery, Union Hospital, Fujian Medical University, Fuzhou, Fujian, 350001, China (E-mail: chenliangwan@tom.com).

J Thorac Cardiovasc Surg 2010;139:1654-5

$0022-5223 / \$ 36.00$

Copyright $@ 2010$ by The American Association for Thoracic Surgery doi:10.1016/j.jtcvs.2009.10.022
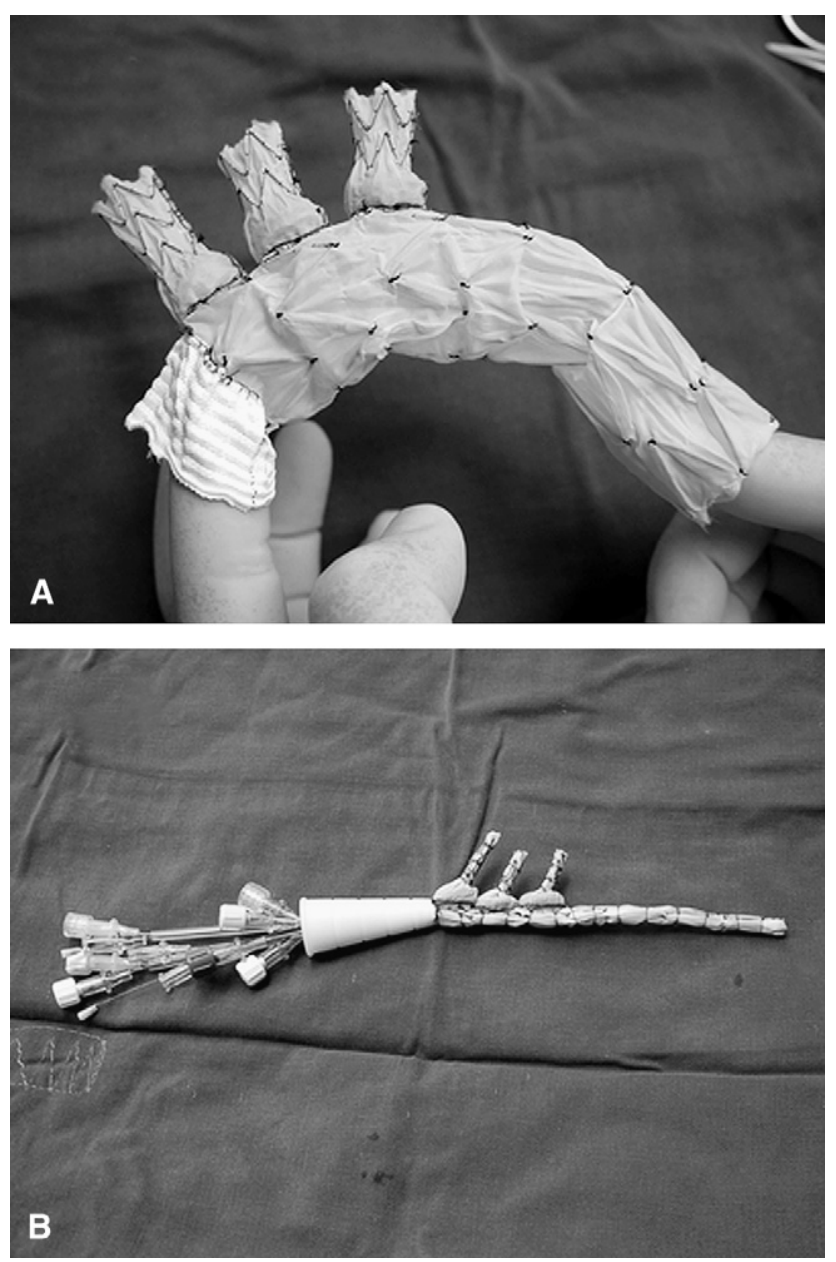

FIGURE 1. The triple-branched stent graft comprised a main graft and three sidearm grafts. It consisted of a self-expandable nitinol stent and polyester vascular graft fabric. The main graft was flexible enough to conform to the curved aortic arch, and at its proximal end, there was a 10-mm long stent-free Dacron tube (A). The main graft and three sidearm grafts were individually mounted on four catheters and restrained by four silk strings (B).

was positioned one by one into the aortic branch. Once the main graft and sidearm grafts were properly positioned, the restraining strings were withdrawn and then the main graft and sidearm grafts were deployed. Finally, the main graft and sidearm grafts were dilated with balloon catheters under the guidance of transesophageal echocardiography to confirm that they were fully opened and not kinked. ${ }^{4}$ The transected stump of the ascending aorta was reconstructed by an inner proximal stent-free Dacron tube of the main graft and outer Teflon felt, and subsequently 


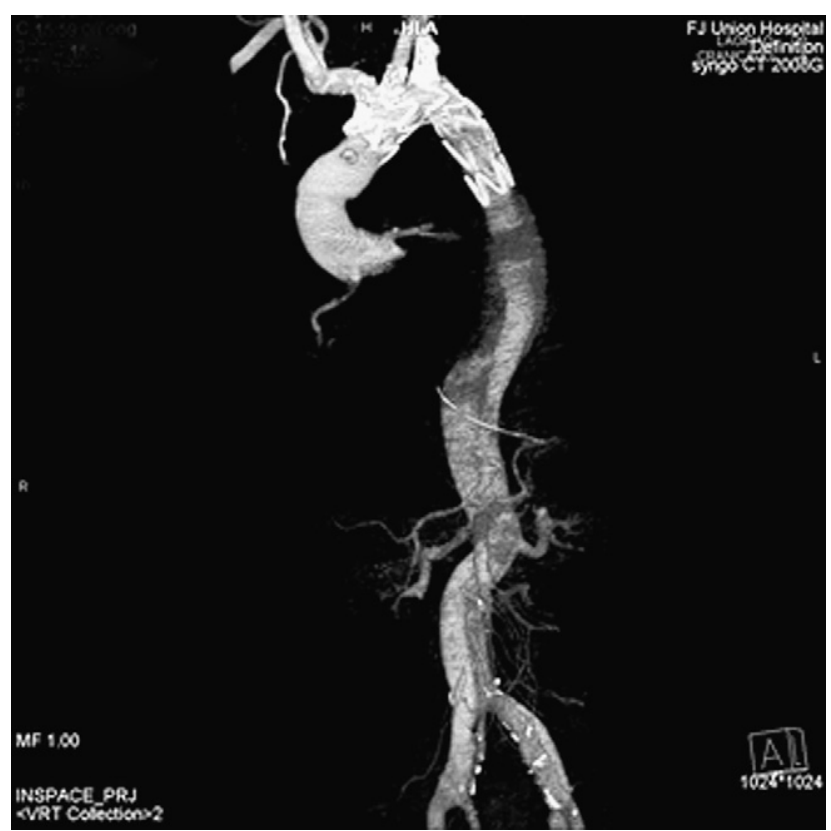

FIGURE 2. Postoperative computed tomographic scans showed that the stent graft was fully opened and not kinked, and there was no space or blood flow surrounding the triple-branched stent graft.

continuous anastomosis to the single-branched Dacron tube graft was made. The air was carefully flushed from the triplebranched stent graft with femoral and right axillary blood return. Then antegrade systemic perfusion from the branch was started and the patient was rewarmed.

The mean cardiopulmonary bypass time, myocardial ischemic time, and open distal time were $155.8 \pm 17.70$, $55.4 \pm 8.50$, and $26.4 \pm 7.33$ minutes, respectively. There were neither postoperative neurologic complications such as coma, paraplegia, and recurrent or phrenic nerve palsy nor malperfusion of organs. All were discharged from the hospital. Their postoperative computed tomographic scans showed that all stent grafts were fully opened and not kinked, and there was no space or blood flow surrounding the triple-branched stent graft (Figure 2). There were no late deaths and no need for reoperation.

\section{DISCUSSION}

By using this triple-branched stent graft, we can avoid the dissection of the arch, anastomosis of the three arch vessels, and the distal anastomosis at the descending aorta. In our practice, we performed the distal aortic anastomosis just at the base of the innominate artery, which provided a much better surgical view than the traditional total arch replacement. Therefore, this open triple-branched stent graft placement technique made total arch reconstruction easier and safer.

For successful triple-branched stent graft placement, diameters of the main graft and three sidearm grafts and the distances between two neighboring sidearm grafts are crucial. The proper size of each graft is key to result in quick clot formation and shrinkage of the false lumen and also to prevent new intimal trauma owing to the continuous compression of the oversized stent graft on the dissected and fragile intimal wall. For each patient, the diameters of the selected grafts should be $10 \%$ bigger than the diameters of the native aortic arch and arch vessels, respectively. ${ }^{1,3,4}$ The distances between two neighboring sidearm grafts should be equal to the distances between two corresponding arch vessels, which would keep sidearm stent grafts untwisted or kinked after their deployment (Figure E1).

In conclusion, open triple-branched stent graft placement is a feasible technique. This technique may make total arch reconstruction easier and safer for the select patient with acute type A aortic dissection. Careful long-term evaluation and many samples of the triple-branched stent graft available will, however, be necessary for extensive clinical use.

\section{References}

1. Kato M, Kuratani T, Kaneko M, Kyo S, Ohnishi K. The results of total arch graft implantation with open stent graft placement for type A aortic dissection. J Thorac Cardiovasc Surg. 2002;124:531-40.

2. Shimamura K, Kuratani T, Matsumiya G, Kato M, Shirakawa Y, Takano H, et al. Long-term results of the open stent-grafting technique for extended aortic arch disease. J Thorac Cardiovasc Surg. 2008;135:1261-9.

3. Flores J, Kunihara T, Shiiya N, Yoshimoto K, Matsuzaki K, Yasuda K. Extensive deploymennt of the stented elephant trunk is associated with an increased risk of spinal cord injury. J Thorac Cardiovasc Surg. 2006;131:336-42.

4. Ishihara H, Uchida N, Yamasaki C, Sakashita M, Kanou M. Extensive primary repair of the thoracic aorta in Stanford type A acute aortic dissection by means of a synthetic vascular graft with a self-expandable stent. $J$ Thorac Cardiovasc Surg. 2002;123:1035-40. 


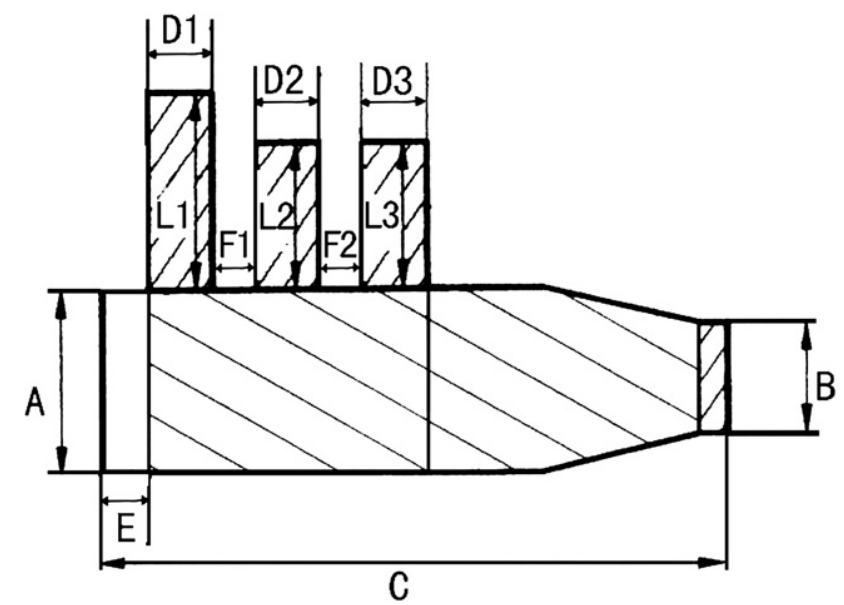

FIGURE E1. The size of the triple-branched stent graft. $A$, The diameter at the proximal end of the main graft, $30 \mathrm{~mm} ; B$, the diameter at the distal end of the main graft, $26 \mathrm{~mm} ; C$, the length of the main graft, $145 \mathrm{~mm} ; E$, the length of the stent-free Dacron tube, $10 \mathrm{~mm} ; D 1$, the diameter of the first side arm graft, $16 \mathrm{~mm} ; D 2$, the diameter of the second side arm graft, $14 \mathrm{~mm} ; D 3$, the diameter of the third side arm graft, $14 \mathrm{~mm} ; L 1$, the length of the first sidearm graft, $35 \mathrm{~mm} ; L 2$, the length of the second sidearm graft, $25 \mathrm{~mm} ; L 3$, the length of the third sidearm graft, $25 \mathrm{~mm} ; F 1$, the distance between first and second sidearm grafts, $3 \mathrm{~mm} ; F 2$, the distance between second and third sidearm grafts, $3 \mathrm{~mm}$. 